J. Clin. Chem. Clin. Biochem.

Vol. 27, 1989, pp. 337-341

(C) 1989 Walter de Gruyter \& Co. Berlin - New York

\title{
An Improved Sensitive Assay Method for the Heterogeneity of $\alpha$-Foetoprotein: Possible Application for Early Differential Diagnosis
}

\author{
By K. Ohkawa and T. Abe
}

Department of Biochemistry, Jikei University School of Medicine

\section{Y. Tsukada}

Biochemical Research Division, Special Reference Laboratory INC.

\author{
K. Taketa
}

Health Research Center, Kagawa University

E. Kimura and Y. Terashima

Department of Obstetrics and Gynaecology, Jikei University School of Medicine, Tokyo, Japan

(Received September 13, 1988/January 16, 1989)

Summary: A combination of lectin-affinity electrophoresis and antibody-affinity blotting was used for the qualitative determination of molecular species of $\alpha$-foetoprotein. Concanavalin A and erythrophytohaemagglutinin were used as lectins. This method was able to quickly discriminate between the molecular species of $\alpha$-foetoprotein of recurrent ovarian yolk sac tumour and those of non-malignant liver diseases and cord sera at term, using only $\overline{3} \mu \mathrm{l}$ of sera containing $200 \mu \mathrm{g} / \mathrm{l}$ of $\alpha$-foetoprotein. The results indicate that this assay method might be useful for the differential diagnosis of recurrent yolk sac tumour from drug- or blood transfusion-induced liver diseases and for the monitoring of the serum $\alpha$-foetoprotein level of patients with yolk sac tumour producing less than $200 \mu \mathrm{g} / \mathrm{l}$ of $\alpha$-foetoprotein.

\section{Introduction}

$\alpha$-Foetoprotein is classified a typical tumour marker for hepatocellular carcinoma and malignant germ cell tumours with yolk sac elements $(1 \div 3)$. In ovarian tumours with yolk sac elements, the elevated serum $\alpha$-foetoprotein level in patients shows a good correlation with the clinical status of malignancy in more than $75 \%$ cases; in addition, an increase in serum $\alpha-$ foetoprotein after a transient decrease due to surgical resection of the tumour is a sign of recurrence $(3-6)$. In the follow-up study after the surgical resection of yolk sac tumours, it is important to determine whether the slight increase of serum $\alpha$-foetoprotein around
$300 \mu \mathrm{g} / \mathrm{l} \mathrm{might} \mathrm{be} \mathrm{due} \mathrm{to} \mathrm{the} \mathrm{recurrence} \mathrm{of} \mathrm{the} \mathrm{tumour}$ or regeneration of the host hepatocytes, after treatment with hepatotoxic anticancer agents or by blood transfusion $(3,7,8)$.

It has been reported that $\alpha$-foetoprotein produced by the yolk sac, foetal liver or regenerating hepatocytes with non-malignant liver diseases can be differentiated by different reactivities against concanavalin $\mathrm{A}$ or erythrophytohaemagglutinin $(9-11)$. This heterogeneity of $\alpha$-foetoprotein with respect to lectin affinity is ascribed to differences in the carbohydrate moiety (12). 
Recently a highly sensitive technique for the detection of small amounts of $\alpha$-foetoprotein was reported by Taketa et al. $(10,13)$. In the present work we applied this rapid and sensitive method to the clinical differential diagnosis of the early stages of recurrent yolk sac tumour from non-malignant liver diseases.

\section{Materials and Methods}

Sera from patients

Frozen samples of sera were obtained from patients with yolk sac tumour without recurrent disease but with hepatic disorders ( 2 cases) and with pathologically confirmed recurrent disease (10 cases, 7 of these with chronic liver diseases indicated by a considerable elevation of serum $\alpha$-foetoprotein from 150 to 500 $\mu \mathrm{g} / \mathrm{l})$. Samples were also collected from patients with nonmalignant liver diseases including liver cirrhosis (30 cases) and from cord sera at term (30 cases).

\section{Determination of serum $\alpha$-foetoprotein level}

Serum $\alpha$-foetoprotein levels were examined using radioimmunoassay (RIA) (14) and Mancini's method (15) with a Japanese $\alpha$-foetoprotein standard (Nippon Bio-Test Lab., Japan) (13) as a reference.

\section{Specific antibody to $\alpha$-foetoprotein}

Specific antiserum against human $\alpha$-foetoprotein was produced in a horse by weekly subcutaneous injections of $1 \mathrm{mg}$ of purified $\alpha$-foetoprotein (16) emulsified in Freund's complete adjuvant. Specific antibody against $\alpha$-foetoprotein was purified by affinity chromatography on $\mathrm{BrCN}$-activated Sepharose 4B (Pharmacia, Sweden) coupled to human $\alpha$-foetoprotein (14).

\section{Isolation of molecular species of $\alpha$-foetoprotein}

$\alpha$-Foetoprotein was fractionated by concanavalin A - or erythrophytohaemagglutinin - affinity agarose gel electrophoresis followed by blotting as described previously $(10,13)$. Briefly, antibody-coated nitrocellulose papers were prepared by incubating with $100 \mathrm{mg} / \mathrm{l}$ affinity-purified horse antibody to human $\alpha$-foetoprotein, then by fixing with glutaraldehyde vapour, neutralizing with $\mathrm{NaBH}_{4}$, followed by washing with Tris-buffered saline $(20 \mathrm{mmol} / 1$ Tris $\mathrm{HCl}, \mathrm{pH} 7.5,500 \mathrm{mmol} / \mathrm{l} \mathrm{NaCl}$, Trisbuffered saline). The nitrocellulose was blocked with $5 \mathrm{~g} / 1$ Tween 20 in Tris-buffered saline for $30 \mathrm{~min}$, then washed with Tris-buffered saline.

Electrophoresis was carried out on $10 \mathrm{~g} / \mathrm{l}$ agarose gel plates (Litex HSA, Litex, Denmark) in barbital/barbital-Na buffer, pH 8.6 containing $1 \mathrm{~g} / 1$ concanavalin A (Sigma, USA) or 0.5 $\mathrm{g} / \mathrm{l}$ erythrophytohaemagglutinin (Sigma, USA) or without lectins. Three microlitres of patients' sera or reference $\alpha$-foetoprotein at various concentrations (diluted with the gel buffer or $\alpha$ foetoprotein-free normal human sera) was applied to the plate and electrophoresis was performed at $4^{\circ} \mathrm{C}$. $\alpha$-Foetoprotein-free sera were prepared by passing sera from healthy subjects through an anti-human $\alpha$-foetoprotein antibody-affinity column. After lectin-affinity electrophoresis, $\alpha$-foetoprotein was transfered, by capillary blotting, to nitrocellulose coated with antibody to $\alpha$-foetoprotein. The nitrocellulose was then washed twice in $0.5 \mathrm{~g} / 1$ Tween 20 in Tris-buffered saline, then treated with rabbit anti-human $\alpha$-foetoprotein IgG (DAKO, Denmark) followed by treatment with swine antibody against rabbit IgG labelled with horseradish peroxidase. Colour was developed by incubating the nitrocellulose with 3,3'-diaminobenzidine tetrahydrochloride (Polyscience, USA) and $\mathrm{H}_{2} \mathrm{O}_{2}$ in Tris-buffered salinc.

\section{Chemicals}

All chemicals were used of reagent grade.

i

\section{Results and Discussion}

Post-operative anticancer chemotherapy with a combination of vincristine, actinomycin $D$ and cyclophosphamide has been employed in patients with recurrent yolk sac tumours. Approximately $70 \%$ of patients treated in this way experienced liver injury, due at least in part to the side effects of the therapy. In these cases serum $\alpha$-foetoprotein levels generally tend to increase. A method to determine at an early stage whether the increase in serum $\alpha$-foetoprotein is due to liver injury or the recurrence of yolk sac tumour (tab. 1) would therefore be clinically advantageous.

The antibody-affinity blotting method showed sensitive results with a detection limit for $\alpha$-foetoprotein of less than $3 \mu \mathrm{l}$ of a solution containing $200 \mu \mathrm{g} / \mathrm{l}$. In the qualitative analysis, $\alpha$-foetoprotein bands could be identified in a sample containing less than $100 \mu \mathrm{g} / 1$ $(300 \mathrm{pg}$ ) of $\alpha$-foetoprotein (fig. 1). By this sensitive method, all the yolk sac tumour $\alpha$-foetoprotein from patients with recurrent yolk sac tumour (10/10) was separated into two bands of concanavalin A-reactive minor and concanavalin A-non-reactive major $\alpha$-foetoprotein (fig. $1 \mathrm{a}$, fig.2). In contrast, all the $\alpha$-foetoprotein obtained from cord sera (30/30) and from the sera of patients with non-malignant liver diseases (30/ 30) showed a single concanavalin A-reactive band (figs. 2,4 ). The same result was also observed in the sera of clinically tumour-free patients with liver injury (data not shown). When erythrophytohaemagglutinin was used as the lectin, all the yolk sac tumour $\alpha$ foetoprotein from the patients with recurrent yolk sac tumours $(10 / 10)$ was fractionated into 3 bands, i.e. strong, weak and non-reactive bands. However, erythrophytohaemagglutinin affinity electrophoresis separated normal $\alpha$-foetoprotein into one or two minor reactive bands and a major non-reactive band (fig. $1 \mathrm{~b}$, fig. 3). One case (case 8 ) indicated co-elevation of yolk sac tumour $\alpha$-foetoprotein and normal $\alpha$ foetoprotein (tab. 1). In this case, $\alpha$-foetoprotein was separated into concanavalin A-reactive and concan-

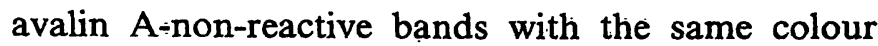
intensity (fig. 4). The patient had clinically been diagnosed as recurrent yolk sac tumour with chronic liver damage, so that the result described above coincided well with the clinical diagnosis. The interre- 


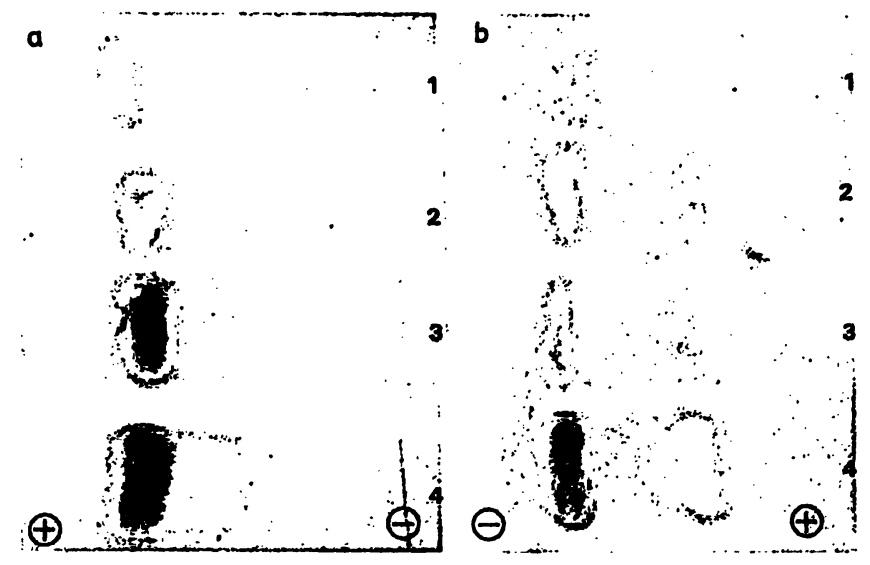

Fig. 1. Sensitivity and typical patterns of antibody-affinity blotting technique for the detection of $\alpha$-foetoprotein separated by agarose gel electrophoresis with concanavalin A (A), erythrophytohaemagglutinin (B). Serum from patient with recurrent yolk sac tumour was serially diluted with $\alpha$-foetoprotein-free serum $(\mathrm{A}, \mathrm{B})$ and $3 \mu \mathrm{l}$ of each sample $(1.100 \mu \mathrm{g} / \mathrm{l}, 2.200$ $\mu \mathrm{g} / \mathrm{l}, 3.500 \mu \mathrm{g} / \mathrm{l}, 4.1000 \mu \mathrm{g} / \mathrm{l})$ was applied. Electrophoresis was run until bromophenol blue migrated $5.0 \mathrm{~cm}$ from the origin.

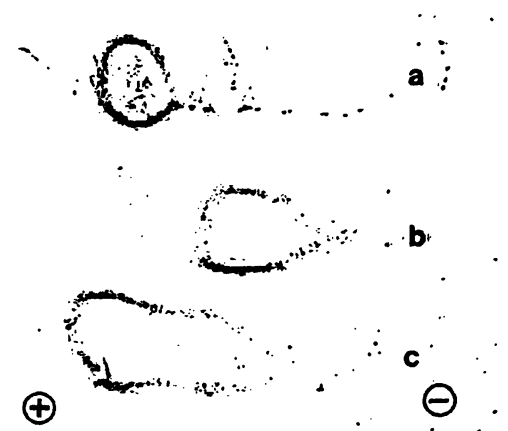

Fig. 2. Typical $\alpha$-foetoprotein (AFP) bands separated by concanavalin A-affinity electrophoresis. Serum from a patient with liver cirrhosis (liver $\alpha$-foetoprotein, $245 \mu \mathrm{g} / \mathrm{l}$, b) and from recurrent yolk sac tumour (yolk sac $\alpha$ foetoprotein; case 9,a; case 4,c) were used. Other conditions of assay, see figure 1. lation between the types of $\alpha$-foetoprotein bands and the pathophysiological status of increased $\alpha$-foetoprotein production is schematically summarized in figure 5 .

Heterogeneous reactivity of $\alpha$-foetoprotein against lectins has been detected by lectin-affinity-crossed immunoelectrophoresis $(9,11,17)$ as well as the combination of lectin-affinity mini-column chromatography with the radioimmunoassay of $\alpha$-foetoprotein (18, 19). However, the sensitivity of the former method for $\alpha$-foetoprotein is around $2 \mathrm{mg} / 1$, unless radiolabeled antibody to $\alpha$-foetoprotein is available. This means that the method described in this paper may have 20-50 times greater sensitivity than that of the former method.

Tab. 1. Clinical data of patients

\begin{tabular}{|c|c|c|c|c|c|c|}
\hline \multirow[t]{2}{*}{ Patient } & \multirow[t]{2}{*}{ Disease } & \multirow{2}{*}{$\begin{array}{l}\text { Blood trans- } \\
\left.\text { fusion }(\mathrm{ml})^{1}\right)\end{array}$} & \multicolumn{4}{|c|}{ Liver function } \\
\hline & & & $\begin{array}{l}\text { Asparate } \\
\text { aminotrans- } \\
\text { ferase }^{2} \text { ) }\end{array}$ & $\begin{array}{l}\text { Alanine } \\
\text { aminotrans- } \\
\text { ferase }^{2} \text { ) }\end{array}$ & $\begin{array}{l}\gamma \text {-Glutamyl } \\
\text { transferase }\end{array}$ & $\alpha$-Foetoprotein $\left.{ }^{2}\right)$ \\
\hline 1 & recurrent $^{3}$ ) & 800 & 50 & 75 & 180 & 350 \\
\hline 2 & reçurrent & 600 & 15 & 8 & 42 & 214 \\
\hline 3 & recurrent & 1500 & 18 & 11 & 40 & 190 \\
\hline 4 & recurrẹt & 2000 & 120 & 95 & 150 & 240 \\
\hline 5 & recurrent & 800 & 54 & 50 & 120 & 217 \\
\hline 6 & recurrent & - & 35 & 25 & 49 & 150 \\
\hline 7 & recurrēent & - & 80 & 43 & 60 & 188 \\
\hline 8 & recurrent & 2000 & 148 & 95 & 110 & 480 \\
\hline 9 & recurren̈t & 2400 & 77 & 65 & 69 & 280 \\
\hline 10 & recurrent & 800 & 54 & 35 & 80 & 297 \\
\hline 11 & no evidence ${ }^{4}$ ) & 3000 & 196 & 130 & 120 & 292 \\
\hline 12 & no evidence & 2200 & 210 & 147 & 98 & 386 \\
\hline
\end{tabular}

1) Blood transfusion was performed either at operation or during post-operative adjuvant chemotherapy.

2) Asparate aminotransferase (reference range 5-40 Karmen units);

Alanine aminotransferase (reference range $0-35$ Karmen units);

$\gamma$-Glutamyl transpeptidase (reference range $0-40 \mathrm{U} / \mathrm{l}$ )

were measured by autoanalyser.

$\alpha$-Foetoprotein (reference range $0-2 \mu \mathrm{g} / \mathrm{l}$ ) was determined with RIA.

3) Clinically and pathologically confirmed recurrent disease.

$\left.{ }^{4}\right)$ Clinically no evidence of disease. 


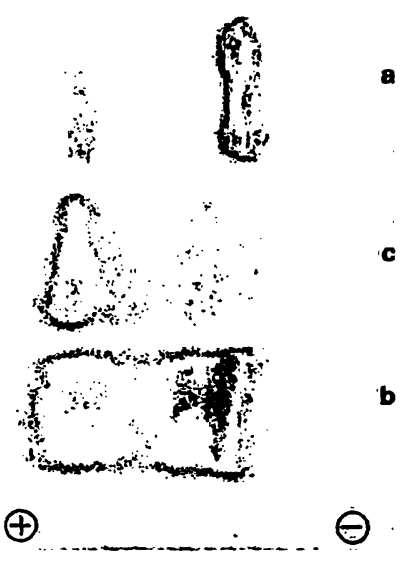

Fig. 3. Typical $\alpha$-foetoprotein (AFP) bands separated by erythrophytohaemagglutinin-affinity electrophoresis. Sera from two recurrent yolk sac tumour (yolk sac $\alpha$-foetoprotein, case 2,a and case 9,b) and cord serum at term (liver $\alpha$-foetoprotein, $190 \mu \mathrm{g} / \mathrm{l}$, c) were used. Other conditions of assay, see figure 1 .

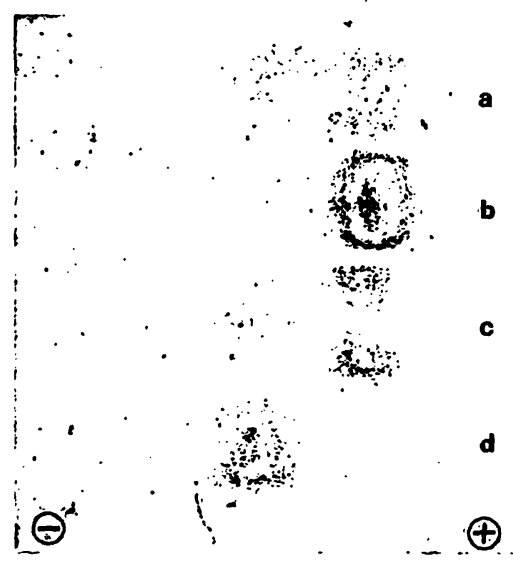

Fig. 4. Analytical $\alpha$-foetoprotein (AFP) bands separated by concanavalin A-affinity electrophoresis. Sera from patients with recurrent yolk sac tumour (case 5,a; case $10, b)$, with recurrent yolk sac tumour with hepatic injury (case 8,c), and with liver cirrhosis (liver $\alpha$-foetoprotein, $250 \mu \mathrm{g} / \mathrm{l}, \mathrm{d})$ were used. In lane c, liver $\alpha$-foetoprotein with the same mobility as lane $d$ was separated from yolk sac $\alpha$-foetoprotein and these two bands indicated the same intensity of colour development suggesting the co-elevation of both types of $\alpha$-foetoprotein.
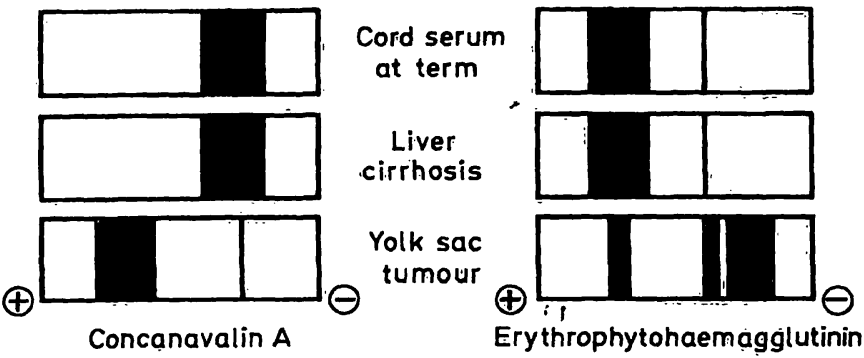

Fig. 5. Schematic representation of $\alpha$-foetoprotein bands separated by affinity-electrophoresis with concanavalin A and erythrophytohaemagglutinin.

As shown by Govindarajan et al. (19), concanavalin A-reactive and non-reactive $\alpha$-foetoprotein fractions in the sera of patients with liver- and germ celltumours can be quantitatively determined in $200 \mu \mathrm{l}$ of serum at $\alpha$-foetoprotein concentrations as low as $58 \mu \mathrm{g} / \mathrm{l}$. This means that the quantitation of bound and non-bound fractions of $\alpha$-foetoprotein by the latter method is clearly superior to the qualitative analysis presented here. But in this latter method it should be noted that serum samples must be appropriately diluted before passing through the concanavalin $\mathrm{A}$ column, to avoid incorrect results due to the presence of other glycoproteins with mannose-type carbohydrate moieties.

Thus, the main advantage of the antibody-affinityblotting method is the fact that very low volumes of serum $(3 \mu \mathrm{l})$ are sufficient for analysis. In comparison with established techniques, the method seems to have a significantly higher sensitivity. Qualitative analysis was based on the identification of the typical reactive patterns of $\alpha$-foetoprotein bands with lectins for $\alpha$ foetoprotein concentrations as low as $100 \mu \mathrm{g} / \mathrm{l}$. This novel method avoids the troublesome manipulation of serum samples, and it is potentially useful for the rapid and early differential diagnosis of recurrent yolk sac tumour and non-malignant liver diseases.

\section{Acknowledgement}

This research was supperted by a Grant-in-Aid for Cancer Research from the Ministry of Education, Science, and Culture, Japan.

\section{References}

1. Gitlin, D., Perricelli, A. \& Gitlin, G. M. (1972) Synthesis of $\alpha$-fetoprotein by liver, yolk sac, and gastrointestinal tract of the human conceptus. Cancer Res. 32, 979-982.

2. Ruoslahti, E. \& Hirai, H. (1978) Alpha-fetoprotein - Purification and its physical and chemical properties. Scand. J. Immunol. 8, Suppl. 8, 3-26.

3. Abelev, G. I. (1971) Alpha-fetoprotein in oncogenesis and its association with malignant tumors. Adv. Cancer Res. $14,295-358$.
4. Wilkinson, E. J., Freidrich, E. G. \& Hosty, T. A. (1973) Alpha-fetoprotein and endodermal sinus tumor of the ovary. Am. J. Obstet. Gynecol. 116, 711-714.

5. Forney, J. P., Disaia, P. J. \& Morrow, C. P. (1975) Endodermal sinus tumor: A report of two sustained remissions treated postoperatively with a combination of actinomycin D, 5-fluorouracil, and cyclophosphamide. Obstet. Gynecol. $45,186-189$. 
6. Kurman, R. J. \& Norris, H. J. (1976) Endodermal sinus tumor of the ovary. Cancer 38, 2404-2419.

7. Nørgaard-Pedersen, B. \& Axelsen, N. H. (1976) Alphafetoprotein-like activity in sera from patients with malignant and non-malignant disease and healthy individuals. Clin. Chim. Acta 71, 343-347.

8. Kai-Li, Xu. (1983) Large-scale AFP screening for hepatocellular carcinoma in China, In: Oncodevelopmental markers, biologic, diagnostic and monitoring aspects (Fishman, W. H., ed.), Academic Press, New York, pp. 395-408.

9. Breborowicz, Mackiewicz, A. \& Breborowicz, D. (1981) Micro-heterogeneity of alpha-fetoprotein in patient serum as demonstrated by lectin affino-electrophoresis. Scand. J. Immunol. 14, 15-20.

10. Taketa, K., Ichikawa, E., Nakabayashi, H., Sato, J., Kato, K., Akai, S., Ohkawa, R., Ohkawa, H., Taga, H. \& Hirai, $H$. (1985) Further resolution of human $\alpha$-foetoprotein by affinity electrophoresis with erythroagglutinating phytohemagglutinin of Phaseolus vulgaris lectin. Tumor Biol. 6, $519-531$.

11. Ishiguro, T., Sugitachi, I., Sakaguchi, H. \& Itani, S. (1985) Serum alpha-fetoprotein subfractions in patients with primary hepatoma or hepatic metastasis of gastric cancer. Cancer 55, 156-159.

12. Ruoslahti, E., Engvall, E., Pekkala, A. \& Seppala, M. (1978) Developmental changes in carbohydrate moiety of human alpha-fetoprotein. Int. J. Cancer 22, 515-520.
13. Taketa, K., Ichikawa, E., Taga, H. \& Hirai, H. (1985) antibody-affinity blotting, a sensitive technique for the detection of $\alpha$-fetoprotein separated by lectin affinity electrophoresis in agarose gels. Electrophoresis 6, 492-497.

14. Nishi, S. \& Hirai, H. (1973) Radioimmunoassay of $\alpha$ fetoprotein in hepatoma, other liver diseases and pregnancy, In: Gann Monogr. 14 (Hirai, H. \& Miyaji, T., eds.), Japan Sci. Soc. Press, Tokyo, pp. 79-82.

15. Mancini, G., Carbonara, A. \& Heremans, J. F. (1965) Immunochemical quantitation of antigens by single radial immunodiffusion. Immunochem. 2, 235-254.

16. Nishi, S. (1970) Isolation and characterization of a human fetal $\alpha$-globulin from the sera of fetuses and a hepatoma patient. Cancer Res. 30, 2507-2513.

17. Bød-Hansen, T. C., Bjerrum, O. J. \& Ramlau, J. (1975) Detection of bispecific interaction during the first dimension electrophoresis in crossed immunoelectrophoresis. Scand. J. Immunol. 4, Suppl. 2, 141-147.

18. Tsuchida, Y., Kaneko, M., Saito, S. \& Endo, Y. (1985) Differences in the structure of alpha-fetoprotein and its clinical use in pediatric surgery. J. Ped. Surg. 20, 260-265.

19. Govindarajan, S., Tse-Ling, F. \& Ashcavai, B. S. (1987) Concanavalin A affinity of alpha-fetoprotein, its use in differentiating tumors. Am. J. Clin. Pathol. 88, 722-724.

\author{
Kiyoshi Ohkawa \\ Dept. Biochemistry \\ Jikei University School of Medicine \\ 3-25-8, Nishi-Shinbashi \\ Minato ku \\ Tokyo \\ Japan
}


